\author{
Marta Pawłowska \\ Uniwersytet Jagielloński w Krakowie \\ m.pawlowska@uj.edu.pl
}

\title{
El léxico de origen hebreo en el judeoespañol contemporáneo
}

\section{Resumen:}

La existencia de un visible componente hebreo constituye una de las características definitorias de las llamadas lenguas judias. El objetivo del presente artículo consiste, por tanto, en determinar el alcance del uso del léxico de origen hebreo en el judeoespañol contemporáneo en su variante escrita. Analizaremos dicho fenómeno basándonos en la prensa sefardí publicada, sobre todo, en Turquía e Israel.

Palabras clave: judeoespañol, hebreo, préstamo, lenguas en contacto

\begin{abstract}
:
Hebrew-derived Lexicon in Contemporary Judeo-Spanish

The presence of a visible Hebrew component is one of the defining traits of the so-called "Jewish languages". The aim of this paper is, therefore, to determine the scope of use of Hebrew-derived lexicon in contemporary Judeo-Spanish in its written variant. Our study will be based on the Sephardic press published mainly in Turkey and Israel.
\end{abstract}

Keywords: Judeo-Spanish, Hebrew, loanword, language contact 
El judeoespañol, al ser una variedad lingüística que durante siglos ha estado expuesta a influencias de varios idiomas de afiliación genética diversa, siempre se ha mostrado propicio a enriquecer su caudal léxico con elementos de procedencia externa. En dichas aportaciones se refleja tanto la fascinante historia de los sefardíes como el carácter innovador de su lengua. En el presente artículo, nuestro interés se centra en el léxico de origen hebreo, dado que por razones religioso-identitarias goza de un estatus particular en comparación con los préstamos de otras lenguas. Además, la mayoría de los hablantes del djudezmo ${ }^{1}$ actualmente residen en Israel o guardan vínculos con este país, lo cual refuerza aún más la posición del hebreo en la comunidad ladinoparlante.

El enfoque que nos planteamos es diferente del de los estudios sefardíes tradicionales, dado que no nos interesa tanto la dimensión diacrónica del djudezmo, sino la descripción sincrónica de su estado actual. Es un campo relativamente poco explorado que, a nuestro parecer, merece una atención especial, en vista de que se trata de una lengua que carece de norma fija y, además, se encuentra en vías de extinción. Todos los estudios del judeoespañol contemporáneo nos parecen, por tanto, particularmente necesarios y valiosos. Siguiendo esta pauta, en el presente artículo nos proponemos llevar a cabo un análisis léxico de la prensa judeoespañola de los años 2013-2015 en función de la presencia y visibilidad en esta del componente hebreo. Pero antes de pasar a dicho examen, haremos un breve repaso de las nociones fundamentales relacionadas con el judeoespañol y, por otra parte, de los conceptos básicos concernientes a los préstamos y términos afines.

\section{El judeoespañol como lengua judía}

En la tradición filológica española, las variedades lingüísticas usadas por los judíos sefardíes, es decir, el ladino (lengua calco utilizada para las traducciones de textos sagrados), el judeoespañol oriental

${ }^{1}$ En el presente artículo, se utiliza el término djudezmo como sinónimo de judeoespañol, siguiendo, entre otros, a Bunis (2011, 2016); Sephiha (1998-99); Weinreich (2008 [1980]) o Wexler (1977). 
y el judeoespañol de Marruecos (llamado haquitía), suelen enumerarse entre los dialectos del castellano - por ejemplo, tienen sus capítulos correspondientes en el Manual de dialectología hispánica de Manuel Álvar (1996) - - y en su estudio se recalcan más bien los vínculos que guardan con el español preclásico. No obstante, si las miramos desde el punto de vista de la historia de las comunidades judías, por ejemplo, al lado de lenguas como el yidis, nuestra óptica cambia, se ponen de relieve otros aspectos y el fenómeno se vuelve más pluridimensional y enriquecedor. Esta idea constituye el fundamento de los trabajos de Max Weinreich:

[W] have to admit that we owe our knowledge of Jewish distinctiveness in the Romanic world of language mainly to representatives of the school of "Judeo-French" for the sake of French, "Judeo-Spanish" for the sake of Spanish, and so on. Assuredly it is scientifically legitimate for them to continue their work. But the time has come to establish the idea that it is legitimate to put the stress on the Jewish specificity also. In the sciences of culture and personality, of which linguistics is one, to detect differences is at any rate as important as perceiving similarities. Looking from within, we may expect more, and not only about Jewish linguistic development; general linguistics also stands to gain more (2008 [1980]: 110).

Siguiendo la misma línea, Weinreich acuñó el término de lengua judía o judeolengua, la cual se distingue de otras variedades lingüísticas por su carácter fusional, es decir, cada lengua judía tiene que ser lengua de fusión, configurada a partir de tres componentes como mínimo. El principal es el componente hebreo que, a pesar de no ser el más importante desde el punto de vista cuantitativo, es el más arcaico en el marco de toda la historia judía. Después, tenemos la lengua anterior de la comunidad (ya enriquecida con elementos hebreos) y la lengua no judía de su entorno. El desarrollo del idioma, que muchas veces lo aleja sustancialmente de la lengua que le sirvió de base, se debe, sobre todo, a influencias mutuas entre los componentes, a interferencias con otras lenguas externas (en particular, en caso de cambiar de territorio) y al desarrollo interno independiente (ibidem, 166-167). Como señala Bunis, 
This kind of linguistic fusion, so characteristic of the Jewish languages of the Diaspora, seems already to be alluded to in the biblical Book of Nehemiah where it is stated that, after the Babylonian exile, the children of Israelites in Jerusalem 'who had married wives of Ashdod, of 'Ammon, and of Moa'v; [...] spoke half in the speech of Ashdod, and could not speak [correctly] in the language of Yehuda, but according to the language of various other peoples' 13: 23-24 (2011: 26).

Obviamente, las lenguas judías difieren en su grado de desarrollo. Por un lado tenemos el yidis y el djudezmo, que se distinguen de manera significativa de sus lenguas de base, y, por el otro, variedades como el judeofrancés o el judeoportugués, en las que estas diferencias resultan escasas. Algunas de estas lenguas cuentan con una producción literaria importante (de nuevo, es el caso del yidis y el djudezmo, pero también del judeoarameo o el judeoárabe), mientras que otras se han documentado tan solo en su variante hablada (por ejemplo, el judeorruso) (Kahn, Rubin, 2016: 3). No obstante, el elemento unificador, presente en todas estas variedades lingüísticas, sea cual fuere su estatus o grado de desarrollo, siempre ha sido el hebreo bíblico (o más bien una mezcla del hebreo y el arameo). Actualmente, la influencia del hebreo moderno también parece significativa, tal y como apuntan Benor y Spolsky: contemporary Jewish languages tend to be influenced by Israeli Hebrew as a result of affiliations with the State of Israel (2006: 121). Así pues, el componente hebreo resulta importante o incluso imprescindible para la configuración de una lengua judía y es uno de sus rasgos definitorios.

Como el judeoespañol cumple todos los requisitos que ha de reunir una lengua judía como tal, también en este idioma los elementos hebreos deberían constituir el núcleo duro. Esta convicción es compartida no solo por los especialistas que se dedican a la llamada lingüística judía, sino también por los mismos hablantes. Zelda Ovadia en uno de sus artículos publicados en Aki Yerushalayim hace referencia a las sienes de palavras ebreas ke uzamos oy en ladino de manera la mas natural ke puede ser (2014). Lo mismo pasa en el artículo de Klara Perahya de la revista Şalom donde el judeoespañol de Turquía se 
define como lenguaje oy en dia heteroklito kon su meskla de palavras de otros idyomas, prioritaryamente del Turko i del Hebreo (2014). Veamos, pues, cuál es realmente el lugar del léxico de origen hebreo en el judeoespañol contemporáneo, pero antes de pasar a dicho análisis repasemos concisamente los fenómenos relacionados con la incorporación en una lengua de vocablos de origen extranjero.

\section{Préstamos: nociones fundamentales}

El concepto de préstamo se puede definir, siguiendo a Haugen, como the attempted reproduction in one language of patterns previously found in another (1950: 212). En función de la fidelidad de dicha reproducción se puede dar el fenómeno de la importación (representación exacta del modelo prestado) o el de la sustitución (su representación inexacta) (ibidem). Ahora bien, estos términos se aplican al procedimiento de préstamo, mientras que en el presente estudio nos centramos en sus resultados; es decir, vamos a someter a análisis los elementos extranjeros, sobre todo léxicos, que ya han entrado en la lengua. Estos extranjerismos varían dependiendo de su estructura y grado de adaptación. En el marco terminológico de Haugen (1950: 213-215), podemos distinguir tres tipos de préstamos léxicos: loanword, hybrid (loanblend) y loantranslation (loanshift), o sea, préstamo propio, híbrido y calco (véase Salamon, 2004: 444), en los que el grado de sustitución morfémica, respectivamente, no se produce, se realiza parcialmente o ha llegado a ser completo. En nuestro caso, un préstamo propio sería, por ejemplo, la palabra mazal 'suerte', mientras que su derivado, mazalozo, formado mediante un sufijo románico, ya constituiría un híbrido. Un claro ejemplo de calco es el verbo suvir que, aparte de 'subir', puede significar también 'emigrar (a Israel)', de la misma manera que su equivalente hebreo תולעל la'alot. Como la cuestión que nos interesa es la visibilidad del componente hebreo en el judeoespañol contemporáneo, haremos especial hincapié en los préstamos propios e híbridos, dejando al margen los calcos.

Al hablar de los préstamos, sería interesante mencionar también el término culturema, empleado en particular en la traductología. Se 
califica de culturema "un elemento verbal o paraverbal que posee una carga cultural específica en una cultura y que al ser transferido a otra cultura, puede provocar una transferencia nula o distinta al original" (Molina Martínez, 2001: 89). A causa de la multiculturalidad de la comunidad sefardí, el trasvase cultural se realiza en el seno de la misma lengua porque los elementos culturales relacionados con el judaísmo y la realidad israelí (vinculados estrechamente con el hebreo) se tienen que expresar en un idioma románico. Por ejemplo, en nuestro corpus encontramos la frase no se djuga kon 'habitakhon' (la seguridad del payis), donde el hablante se sirve de un hebraísmo perfectamente traducible al judeoespañol (de hecho, se nos ofrece dicha traducción), pero, a pesar de que el significado denotativo resulta el mismo, en este contexto el préstamo funciona a modo de culturema aportando un matiz diferente a toda la expresión.

\section{El léxico judeoespañol: información general}

La naturaleza de las lenguas judías y los mecanismos de su desarrollo ya nos indican que el préstamo será un fenómeno muy frecuente en este tipo de idiomas. En nuestro caso, todos los componentes que han influido en la configuración del djudezmo han dejado huellas en su léxico. Así pues, aparte del vocabulario perteneciente al español preclásico, encontraremos elementos procedentes de otras lenguas peninsulares (portugués, catalán, aragonés, etc.), de otras lenguas románicas (italiano y, especialmente, francés), de lenguas balcánicas (búlgaro, griego, etc., en función de la zona), del turco, del hebreo $\mathrm{y}$, últimamente, del inglés (para ejemplos, véase, entre otros, Bunis (2011), Díaz Mas (1989), Larm Jallow (2007), Peramos Soler (2010), Quintana (2001), Sala (1999)). Según Sephiha, el judeoespañol como lengua de fusión "comprend 4\% d'emprunts à l'hébreu, 15\% au turc, $20 \%$ au français, $1 \%$ au grec, $2 \%$ au ladino, etc., le tout sur un substrat espagnol du XVème siècle" (1997: sin página).

Centrémonos ahora en el componente hebreo. En los estudios que se han llevado a cabo se indica que la influencia hebrea se ha producido, en mayor o menor grado, en todos los niveles del idioma. Desde 
el punto de vista fonológico, se puede mencionar la aparición de los fonemas $/ \mathrm{ts} / \mathrm{y} / \mathrm{x} /$ en préstamos (aunque esta no se debe exclusivamente al hebreo, sino también a otras lenguas con las que el djudezmo ha entrado en contacto). En cuanto a la morfología, por ejemplo, llama la atención la introducción en el sistema flexivo de las desinencias hebreas de plural: -im para el masculino y -ot para el femenino (principalmente en hebraísmos, aunque -im se da de forma ocasional en algunas palabras de origen español o incluso turco). En el ámbito de la sintaxis también sería posible indicar ciertas construcciones propias del hebreo (véase, por ejemplo, Sala (1999)). No obstante, sin lugar a dudas es el nivel léxico el que se ha enriquecido más con elementos de procedencia hebrea (o hebreo-aramea).

Entre las áreas temáticas más propicias a absorber hebraísmos, obviamente se contarán los términos relacionados con el judaísmo, es decir, del léxico religioso. Además, se puede destacar el vocabulario para expresar nociones abstractas. Según los investigadores, estos dos grupos se atestiguan ya en los textos sefardíes anteriores a la expulsión. En el siglo XVI, el hebreo se convierte en una fuente importante de palabras vinculadas con las emociones (estados psicológicos), de eufemismos para lo considerado tabú y de nombres de otras religiones o conversiones religiosas. Asimismo, se importan algunos vocablos anatómico-médicos (a veces arraigados en la medicina popular y el ocultismo) y términos de la vida cotidiana, sobre todo del ámbito de la familia y del comercio. Cabe mencionar que entre los siglos XVI y XX se puede documentar una alternancia de uso entre numerosos hebraísmos y sus sinónimos de origen hispánico. No obstante, el empleo de vocablos de procedencia hebrea disminuye a principios del siglo XX debido a las tendencias asimiladoras surgidas de la haskalá (Bunis, 2016: 406-410).

\section{Los hebraísmos léxicos en la prensa judeoespañola: análisis del corpus}

A fin de determinar cuál es la presencia real de los hebraísmos en el judeoespañol contemporáneo, hemos analizado artículos publicados 
en la prensa sefardí entre los años 2013-2015. Las dos fuentes principales han sido la revista Aki Yerushalayim, publicada en Israel, y la página en djudezmo del semanario Şalom, de Turquía. Los textos de Şalom fueron escritos por más de diez autores, todos hablantes nativos, mientras que en Aki Yerushalayim contamos con más de dieciséis autores, mayoritariamente hablantes nativos, aunque también se publican textos traducidos. El corpus consta de 396041 ocurrencias (en calidad de ocurrencias se cuentan tanto palabras como signos de puntuación), entre las que se pueden distinguir 34305 tokens diferentes ${ }^{2}$. La mayor parte del material estudiado proviene de Şalom (318 816 ocurrencias, 29175 tokens), mientras que Aki Yerushalayim presenta 77225 ocurrencias (lo que se traduce en 10262 tokens). Aparte del corpus principal, como fuente de ejemplos también nos ha servido la revista venezolana Maguen Escudo (1 781 ocurrencias, 756 tokens) ${ }^{3}$. Debido a su escasez y carácter particular, no se analizan artículos escritos en haquitía. Así pues, la recopilación de textos refleja el fenómeno en cuestión esencialmente en el judeoespañol de Turquía, lo cual nos parece especialmente interesante, en vista de que los sefardíes de este país son descendientes directos de las comunidades judías del antiguo Imperio otomano y, además, su judeoespañol estará menos influido por el hebreo moderno que el de los hablantes asentados, ya desde hace tiempo, en el Estado de Israel.

\subsection{Observaciones preliminares}

Antes de todo, nos parece lícito enumerar ciertos fenómenos presentes en el judeoespañol que se vinculan directamente a las influencias judías. En primer lugar, se suelen incorporar expresiones o incluso frases enteras en hebreo. En la mayoría de los casos se trata de deseos y bendiciones (Shana Tova 'buen año', Hag Sameah 'feliz fiesta', a veces con el nombre de la fiesta añadido: Hag Pesah Sameah, Hag Shavuot

${ }^{2}$ Cada forma registrada constituye un token, incluyendo los signos de puntuación. No se trata de un corpus anotado; así pues, el número de tokens no corresponde al número de lemas.

3 Maguen Escudo se publica mayoritariamente en castellano con algunos artículos en judeoespañol. 
Sameah, etc., Tizku Leshanim Rabot 'que merezcas muchos años', Azlaha i Beraha 'éxito y bendición', Baruch Hashem 'bendito sea el nombre'), pero también aparecen citas de oraciones o textos de índole religiosa (por ejemplo, Ma nishtanah ha layla haze mi kol haleilot? ‘Por qué la noche de hoy es diferente de las demás noches? ’: pregunta que inaugura la hagadá de Pésaj). Desde un punto de vista formal, ya en estos ejemplos se puede ver una cierta vacilación en cuanto al uso de mayúsculas y minúsculas, oposición ortográfica desconocida en los alfabetos semíticos. El empleo excesivo de mayúsculas puede explicarse por motivos religiosos (para marcar respeto) o también por la influencia de otras lenguas habladas por los ladinófonos (turco, inglés, francés, etc.).

Además, una tendencia bastante visible consiste en el uso paralelo de palabras o expresiones de origen hebreo junto con sus equivalentes románicos. Por un lado, se trata de aclaraciones, normalmente en los casos de traducciones al djudezmo de conceptos propios del judaísmo. Lo podemos apreciar en los siguientes ejemplos, en los que los vocablos de uso general se concretan mediante hebraísmos y, de este modo, se inscriben directamente en el contexto judío: Tribunal Rabiniko (Beit Din); resivio su graduasion (semiha) de rabino; la Vieja Komunidad (Yishuv) sefaradí. A veces, nos encontramos con situaciones en las que el hebraísmo simplemente complementa el texto: Elul es konosido komo mez de piadad: "Hodesh Harahamim". No obstante, también se dan casos curiosos: por ejemplo, en uno de los textos la palabra hebrea que sirve de aclaración en realidad coincide con la española, mientras que el djudezmo recurre a un galicismo: sovre la karta jeografika (komo dizen en ebreo "hamapa").

Por otro lado, si bien se suelen concretar o aclarar los vocablos románicos traduciéndolos al hebreo, la tendencia opuesta resulta mucho más frecuente, es decir, los hebraísmos también se traducen o se tienen que explicar. Este procedimiento se aplica, sobre todo, a la esfera de la religión: un Sefer Tora (rolo de la Tora); un perush (komentario) a la Mishna; los "sidurim" (livros de orasion); un mahzor (livro de orasiones) espesial; los tefilim (filakterias); la Geula, la Salvasión; dos "Talmud Tora" o eskolas religiosas; asegun la alaha, las prinsipalas 
leyes djudias; kale dizir la beraha de ,a-tov ve-ametiv', kere dizir, El ke es bueno i ke aze bueno'; El mez de Adar es prezentado al puevlo Djudio, komo "Marbin Besimha" (pujar la alegriya); Pesah es referado komo "Zman Sheirateynu”. Kere dezir: el momento de muestra delivrensa i de libertad; A las vezes, esta fiesta, la yaman Zeman Simkhateinu (Sezon de Alegriya); piyutim, poemas liturjikos; maftirim, ke es uno de los djeneros autentikos de la liturjia djudia; pero también a fenómenos históricos o sociales, relacionados obviamente con la historia judía o el Estado de Israel: los "anusim"- termino adoptado ultimamente para los ke se uzava yamar, de antes, "marranos" o "djudios sekretos"; los B'nai Anusim (marranos); los maridos no keren darles el 'get' (el dokumento relijiozo ke permete el divorso); En el pasado, una aguna era una mujer ke el marido se fue a la gerra, i no torno mas atrás; no se djuga kon 'habitakhon' (la seguridad del payis); la Primera Aliya (primera onda de olim / imigrantes); las primeras Aliyot [ondas de imigrasion sionista a la Tierra de Israel]. En este caso, se trata más bien de palabras que describen conceptos muy concretos, muchas veces sin equivalentes.

\subsection{Principales áreas temáticas}

Pasemos pues a los hebraísmos que se utilizan ya de manera independiente, sin necesidad de traducción. Empecemos por los nombres propios. No es de extrañar que los sefardíes se sirvan de topónimos o antropónimos en su forma bíblica, a pesar de que todas estas palabras tienen sus adaptaciones románicas al pertenecer al acervo cultural judeocristiano. Tenemos, por tanto, Yerushalayim en vez de Jerusalén (y sus gentilicios serán yerushalmi o yerushalmita) o Kenaan por $\mathrm{Ca}$ naán. A veces el mismo lugar puede recibir dos nombres en función del significado: es el caso de Egipto para el que, al lado de vocablos románicos de tipo Ejipto o Ayifto, tenemos el topónimo de origen hebreo, Misraim, que suele utilizarse en el contexto bíblico (como para referirse, por ejemplo, a la salida de los judíos de Egipto), pero no exclusivamente. Otros nombres de lugares que suelen aparecer en versión hebrea son el Bet 'Hamikdash 'el Templo de Jerusalén', Erets Israel 'la Tierra de Israel' y el Kotel 'Hamaaravi o el Kotel Maaravi 
'el Muro de las Lamentaciones', literalmente 'el muro occidental'. Si analizamos este último ejemplo desde el punto de vista morfológico, detectamos una vacilación en cuanto a la presencia del artículo definido hebreo $\mathrm{B} / \mathrm{ha} /$ en los préstamos. En cuanto a los antropónimos, también se trata de nombres bíblicos: Moshe Rabenu 'Moisés, nuestro maestro', David Ha Meleh 'el rey David', Shelomo Ha Meleh 'el rey Salomón' y Ester Malka 'reina Ester'. No obstante, la cuestión del artículo vuelve a manifestarse. Parece curioso que los nombres de los reyes reproduzcan su forma original con el determinante, mientras que el nombre de Ester carezca del $h a$, aunque este también debería presentarse siguiendo el patrón morfosintáctico hebreo.

Al tratar el tema de los nombres propios, es preciso abordar la cuestión de los nombres de Dios o, en la mayoría de los casos, de las perífrasis generalizadas acuñadas para no tomar el nombre de Dios en vano. En este contexto, nos encontramos con cuatro procedimientos: deformación ortográfica de la palabra para 'dios' u otras afines (precisamente para evitar nombrar a Dios), nombres en hebreo, calcos de las denominaciones hebreas y expresiones híbridas. En el primer grupo se pueden citar los siguientes ejemplos: D.; D.yo; Di-o; D. Yeuda. Cabe añadir que la forma dio sin la $-s$ final se remonta aún a los tiempos anteriores a la expulsión. Los judíos interpretaron la $-s$ como marca del plural, de ahí que la eliminaran porque a su parecer se asociaba con el politeísmo. En cuanto a los nombres hebreos, tenemos Ado-nay (también con cierta deformación en la grafía), que significa 'mi señor' y también suele usarse en la religión cristiana, Ribono Shel Olam 'señor del mundo' o Hakadosh Baruh Hu 'el santo y bendito él'. Esta última denominación aparece también traducida como el Santo $i$ Bendicho El. Otros calcos serían el Kriador Bendicho El y el "Bendicho sea su Nombre", lo que en hebreo es משה דורב Baruh HaShem, que hoy en día se utiliza en Israel incluso a modo de saludo. Como híbrida se puede interpretar la expresión el Dyo Baruhu 'dios bendito', que se inscribe bien en toda esta serie de nombres perifrásticos.

En el apartado de los nombres propios se pueden incluir también los de fiestas y celebraciones judías, así como los títulos de ciertos libros santos, oraciones, etc. Nos encontramos, por tanto, con toda 
una serie de festividades propias del judaísmo: Yamim Noraim, Rosh Hashana, Yom Kipur (también en la versión Yom Ha Kipurim o en la forma híbrida Dia de Kipur, puesto que la palabra פוי yom significa 'día'), Purim, Pesah, Seder, Lag Baomer, Shavuot, Hag Habikurim, Sukot, Shemini Atseret, Simhat Tora, Hanuka, Tisha BeAv, Tu Bishvat, Taanit de Ester, Hag Hamatzot, Taanit de Shiva Asar be Tamuz o fiestas del Estado de Israel como Yom Hashoa 'Día del recuerdo del Holocausto', Yom Yerushalaim, Yom Hatsmaut 'Día de la independencia'. También, obviamente, se mencionan celebraciones tales como Bar Mitsva (en realidad, el origen de esta construcción es arameo; en hebreo debería ser baal mitsva) o Bat Mitsva. Además, ya como nombres comunes se utilizan palabras hebreas como hag 'fiesta', yom tov 'buen día', también equivalente de 'fiesta', y taanit 'día de ayuno'. Tampoco es de extrañar que en lo que concierne a fiestas se usen nombres de meses del calendario judío. Así pues, en el corpus los tenemos casi todos: Tishri, Heshvan, Tevet, Shevat, Adar, Nissan, Iyar, Sivan, Ta$m u z, A v$, Elul (faltaría solo Kislev). También se usan otros vocablos relacionados con el calendario y el cómputo de días: evidentemente a menudo aparece shabat, pero asimismo se documentan expresiones como motsae shabat 'la salida del shabat', rosh hodesh 'primer día del mes', literalmente 'cabeza del mes', y sefirat omer 'la cuenta del Omer', período entre Pésaj y Shavuot. Es interesante observar que en este último ejemplo de nuevo se ha omitido el artículo definido, ya que debería ser Sefirat Ha'omer.

En lo que concierne a los títulos, se trata, sobre todo, de las denominaciones de los libros santos (o sus partes) y las recopilaciones de reglas religiosas. En el corpus se citan: Halaha, Tanah, Tora, Sefer Tora 'un rollo de la Torá', Megila de Ruth 'Libro de Rut', Megilla Ester 'Libro de Ester', Talmud, Mishna, Mishne Tora. También se mencionan títulos de oraciones o plegarias: Shemá Yisrael y Kol Nidrei. En este grupo podemos incluir asimismo el nombre Kabala.

Ya con estos ejemplos se puede ver que el área temática de la religión tendrá el mayor peso y la mayor representación entre todos los hebraísmos detectados en el corpus. Veamos estos vocablos, clasificados en grupos: 
- oraciones, bendiciones, cantos, etc.: beraha 'bendición', sheva berajot 'siete bendiciones' (bendiciones de la boda), selihot 'perdones' (poemas y oraciones penitenciales), hallel 'alabanza' (tipo de oración basado en los salmos), tefila 'oración', minha (oración de la tarde), arvit (oración de la noche), amen, aleluya;

- géneros tradicionales, unidades textuales, etc.: teilim 'salmos', perasha (porción de la Torá), perush 'comentario', hagada 'narración', piyutim 'poemas litúrgicos';

- prácticas religiosas, actos rituales, etc.: taanit 'ayuno', teshuva 'arrepentimiento', neder 'voto' (promesa solemne a Dios), tashlih 'echar' (rito de echar los pecados simbólicamente), berith mila / berit 'circuncisión', sedaka / tsedaka 'caridad, limosna' (y su derivado: sedakero);

- alimentos y comidas: seuda (comida festiva), hallah 'jalá', matza, hamets (masa madre), maror (hierba amarga), kashrut, kosher / kasher;

- sinagogas y sus partes y otros espacios de culto, etc.: kal 'sinagoga', keila 'sinagoga', azara (espacio de mujeres), bima (plataforma desde la cual se leen los rollos de la Torá), teva (sinónimo de bima), aron 'hakodesh (armario donde se guardan los rollos de la Torá), ehal akodesh (sinónimo de aron 'hakodesh), parohot (cortinas que cubren las puertas del Arón Ha-Kódesh), mikve (espacio donde se realizan los baños de purificación), betahaim 'cementerio';

- objetos litúrgicos o afines: hupa (palio nupcial), mezuza (pergamino con dos versículos de la Torá colocado a la entrada de la casa), menora (candelabro de siete brazos), hanukia (candelabro de nueve brazos usado en la fiesta de Januká), shamash (vela auxiliar de la januquiá), suk(k)a 'tabernáculo' (lugar de residencia temporal construido para fiesta de Sukkot), shofar (instrumento musical utilizado durante algunas festividades), tefilin 'filacteria';

- cargos, oficios y otras denominaciones referidas a personas: el Koen Agadol 'sumo sacerdote', rav, rabi, ribi, rabino, haham 'rabino' (lit. 'sabio', la misma palabra significa 'rabino' en la lengua turca), hazan (oficiante que guía los cantos en la sinagoga), 
yahid (miembro de la comunidad, fiel de la sinagoga), ma'aminim 'creyentes';

- corrientes del judaísmo: haredim (ultraortodoxos), chabad (un movimiento ortodoxo jasídico), karay (judaísmo caraíta);

- vestimenta: kipa (gorra ritual), tallet 'talit';

- otros: emuna 'fe', lashon (lashon ha-kodesh) 'lengua (lengua sagrada)' (sobre todo, en referencia al hebreo), Mashiah 'mesías', Eden, ganeden (lit. jardín del Edén) 'paraíso', gueynam 'infierno'.

Es interesante notar que en este vocabulario han penetrado palabras y expresiones de origen askenazí, aunque se documentan también sus equivalentes sefardíes. Tenemos, por tanto, kosher al lado de kasher o bima y aron 'hakodesh junto a teva y ehal akodesh.

Otra palabra que llama nuestra atención y merece un comentario es betahaim 'cementerio'. Se trata de un vocablo acuñado según un esquema muy productivo en hebreo en el que la palabra 'casa' forma un status constructus con algún otro sustantivo. En nuestro caso, betahaim significaría literalmente 'la casa de la vida' y es una invención sefardí.

La parte esencial del corpus referida a lo sagrado que acabamos de ver se puede completar con vocablos de áreas temáticas que ya no pertenecen a la esfera del culto propiamente dicha, pero que están estrechamente unidas al judaísmo. Así pues, nos encontramos con nombres de escuelas religiosas o lugares de estudio: Talmud Tora, yeshiva y bet midrash; y con términos del ámbito jurídico (obviamente, se trata de la ley judía): mitzva 'mandamiento', bet din 'tribunal rabínico', ketuba 'contrato matrimonial', aguna (mujer cuyo marido no puede o no quiere divorciarse de ella), get 'divorcio'.

La siguiente área semántica engloba vocablos relacionados con la historia judía. Se trata de términos generales como galut 'destierro, diáspora', goy 'no judío', Yishuv 'asentamiento (en la Tierra de Israel)', denominaciones de grupos de judíos: anusim / beneanusim 'marranos' o los Beta Israel 'falashas' (judíos de origen etíope); o expresiones referidas al Holocausto: la Shoa, Magen David 'estrella de David' (lit. 'escudo de David'). También se usan palabras vinculadas a la realidad israelí de tipo kibbutz (comuna agrícola israelí), moshav 
(comunidad rural de carácter cooperativo), la K(e)neset (el parlamento de Israel), Tsahal 'Fuerzas de Defensa de Israel' o shekalim 'siclos'. Un ejemplo interesante lo ofrecen el sustantivo aliya y sus derivados. Esta palabra literalmente significa 'subida' y designa la emigración judía a la Tierra de Israel. A este tipo de emigrante se lo llama en hebreo שישדח םילוע olim jadashim, donde jadash, en plural שדח הלוע olade el adjetivo שדח jadash significa 'nuevo'. Así pues, en judeoespañol se documentan formas como los olim, parcialmente traducida como los muevos olim o los ole hadashim, donde solo el último miembro del sintagma recibe la marca del plural. La existencia de la palabra aliya influyó también en la ya mencionada ampliación del significado del verbo suvir como 'emigrar': suvio a Israel en 1949.

En cuanto al vocabulario más cotidiano, encontramos vocablos del ámbito familiar como mishpaha 'familia', avot 'padres', balabay 'dueño de la casa', nociones abstractas de tipo mazal 'suerte' (y sus derivados mazalozo y dezmazalado), hen 'encanto', sehel 'inteligencia', azlaha 'éxito, abundancia', ahadut 'unidad', parnasa 'ganancia', zehut / zahú 'mérito', yesurin 'sufrimientos', heshbon 'cálculo' y otras palabras sueltas: benadam 'hombre, ser humano', olam 'mundo', aver 'aire', erev 'tarde, atardecer', malsin 'delator', madrih 'guía', bet zekenim 'residencia de ancianos'. Se han atestiguado también dos sustantivos híbridos: uno hebreo-turco purimlik 'regalo de purim' y otro ya español-hebreo eskupesehel 'sabelotodo'.

Como acabamos de ver, estos préstamos casi en su totalidad son sustantivos. Cuando aparece algún adjetivo, suele ser en realidad un híbrido formado a base de un nombre hebreo mediante morfemas derivativos románicos. Los adjetivos en su forma hebrea o se usan en expresiones fijas, o sea, acompañando nombres, o funcionan como sustantivos (haham, haredim). Las únicas excepciones son kosher / kasher y karay. Las demás partes de la oración casi no se atestiguan en el corpus. Se puede mencionar tan solo la preposición $m i$ 'de' en el nombre propio el Rabino Binyamin mi Tudela y el adverbio afilu 'incluso'. Además, se suele emplear con frecuencia la sigla: $z$ "l (pronunciada zal o en su forma plena zijronó li-vrajá) ‘de bendita memoria, lit. 
su memoria a la bendición', aunque también se utiliza su equivalente románico de b.m.

\section{Conclusiones}

El caudal léxico de origen hebreo documentado en la prensa judeoespañola contemporánea en la mayoría de los casos constituye simplemente un vocabulario compartido por todos los judíos, en vista de que se trata de términos cuyos referentes se vinculan estrechamente con la religión y cultura judía. Por la misma razón, una parte considerable de dichos hebraísmos, que podemos calificar de culturemas, funcionan también en otras lenguas.

En segundo lugar, es preciso subrayar que la frecuencia de apariciones de las palabras analizadas resulta muy baja. En Aki Yerushalayim, el hebraísmo más frecuente (excluyendo topónimos y antropónimos) es el sustantivo $\mathrm{kal}$ 'sinagoga', documentado 50 veces, seguido por rabino, que en realidad ya es una adaptación románica, que coincide con la forma que se usa en castellano (30 veces), el título Tora (23 veces) y el vocablo anusim (también 23 veces, siempre en plural). En Şalom, es decir, un corpus casi cuatro veces mayor, entre las palabras más usadas tenemos denominaciones de fiestas: Pesah (122), Purim (61), Hanuka (60); obviamente también aparece Tora (87) y rabino (96). El primer sustantivo común que podemos considerar hebraísmo puro es rav, equivalente hebreo de rabino, que se documenta 69 veces.

Desde el punto de vista de la adaptación, estos elementos se suelen adaptar fonéticamente (mediante inserción de vocales de apoyo, sustitución de la consonante /ts/ por /s/, etc.), pero a veces resultan poco integrados en el sistema morfológico y mal interpretados por los hablantes, lo que concierne sobre todo a los mencionados sintagmas en status constructus o a la cuestión del artículo hebreo, pero también a las vacilaciones en el género o en la formación del plural.

Así pues, nos encontramos ante un fenómeno fosilizado y, en realidad, de poco alcance, pero que al mismo tiempo es visible y de un claro valor simbólico e identitario. No obstante, hay que tener en cuenta que la visión que obtenemos se debe, hasta cierto punto, al 
carácter particular de la prensa sefardí. Los textos analizados pueden sorprender no solo por el relativamente escaso número de hebraísmos, sino también por la ínfima presencia de turquismos, cosa que parece incluso más impactante, dado que en la mayoría de los casos se trata de autores turcos o de procedencia turca. Este fenómeno se puede explicar por una cierta tendencia a aclarar la exposición (algo que hemos visto ya en una serie de ejemplos hebreos). Además, en algunos textos de Şalom se lleva a cabo un proceso que podemos denominar autocensura. Los autores evitan conscientemente el uso de vocablos de procedencia turca en un intento por velar por la pureza del lenguaje, pero a causa de su desconocimiento del castellano moderno cubren las lagunas léxicas con préstamos del francés. A pesar de que los hebraísmos gozan de mucho más prestigio que los turquismos, debido a su dimensión ideológico-religiosa, podemos suponer que los sefardíes no residentes en Israel no recurren al vocabulario de este origen, dado que no cuentan con un nivel lingüístico suficiente fuera de los ámbitos tradicionales relacionados con el judaísmo para que dicha lengua pueda constituir una fuente importante de préstamos recientes. No obstante, este componente sigue estando presente en el idioma y, sin duda alguna, es una de las marcas de su particularidad.

\section{Bibliografía}

ÁLVAR, M. (ed.) (1999), Manual de dialectología hispánica. El Español de España, Ariel Lingüística, Barcelona.

BENOR, S. B., SPOLSKY, B. (2006), “Jewish Languages”, en: Brown, K. (ed.), Encyclopedia of Language \& Linguistics, Elsevier, Oxford, vol. 6, pp. 120-124, [on-line] https://doi.org/10.1016/B0-08-044854-2/04397-2. BUNIS, D. (2011), "Judezmo: The Jewish Language of the Ottoman Sephardim", European Judaism. A Journal for the New Europe, 44(1), pp. 22-35. BUNIS, D. (2016), "Judezmo (Ladino)”, en: Kahn, L., Rubin, A. (eds.), Handbook of Jewish Languages, vol. 2, Brill, Leiden, pp. 365-450, [on-line] https://doi.org/10.1163/9789004297357_014. 
DÍAZ-MAS, P. (1989), "Influencias francesas en la literatura sefardí: estado de la cuestión”, en: Lafarga, F. (ed.), Imágenes de Francia en las letras hispánicas, Promociones y Publicaciones Universitarias, Barcelona.

HAUGEN, E. (1950), "The Analysis of Linguistic Borrowing”, Language, 26(2), pp. 210-231, [on-line] https://doi.org/10.2307/410058.

KAHN, L., RUBIN, A. (eds.), Handbook of Jewish Languages, Brill, Leiden, [on-line] https://doi.org/10.1163/9789004297357.

LARM JALLOW, M. (2007), Préstamos léxicos en el judeo-español contemporáneo en «De Saragosa a Yerushaláyim», Göteborgs Universitet, Göteborg.

MOLINA MARTÍNEZ, L. (2001), Análisis descriptivo de la traducción de los culturemas árabe-español, Universitat Autònoma de Barcelona, Barcelona.

OVADIA, Z. (2014), "La enfluensa del turko sovre el djudeo-espanyol”, Aki Yerushalayim. Revista Kulturala Djudeo-espanyola, 95.

PERAHYA, K. (2014), "Parcours Judeo-Espagnol et patrimoine en Méditerranée", SSalom,1.10, [on-line] http://www.salom.com.tr/haber-92545-parcours_judeoespagnol_et_patrimoine_en_mditerrane.html?rev=2, 22.08.2017.

PERAMOS SOLER, N. (2010), El judeo-español en Salónica. Influencias lingüisticas, Servicio de publicaciones: Universidad de La Laguna, La Laguna.

QUINTANA, A. (2001), “Concomitancias lingüísticas entre el aragonés y el ladino (judeoespañol)", Archivo de Filología Aragonesa, 57-58, pp. 163-192.

SALA, M. (1999), "El judeo-español balcánico", en: Álvar, M. (ed.), Manual de dialectología hispánica. El Español de España, Ariel Lingüística, Barcelona, pp. 360-367.

SALAMON, M. (2004), “Aspectos léxico-semánticos de los verbos prestados del inglés”, Studia Romanica Posnaniensia, 31, pp. 443-449, [on-line] http://dx.doi.org/10.14746/strop.2004.31.044

SEPHIHA, H. V. (1997), "Le judéo-espagnol” en: Sephiha, H. V., Weinstock, N., Yiddish et judéo-espagnol. Un héritage européen, Bureau Européen pour les Langues Moins Répandues, Bruxelles. 
SEPHIHA, H. V. (1998-99), "Langue et littérature judéo-espagnoles", Plurielles, 7, Hiver-Printemps, pp. 70-77.

WEINREICH, M. (2008 [1980]), History of the Yiddish Language, vol. 1, Yale University Press, New Haven/London.

WEXLER, P. (1977), "Ascertaining the position of Judezmo within Ibero-Romance", Vox Romanica, 36, pp. 162-195, [on-line] http://doi.org/10.5169/ seals-28582

\section{Páginas web (Corpus)}

https://revistamaguenescudo.wordpress.com/, 22.08.2017

http://www.aki-yerushalayim.co.il/ay/anteriores.htm, 22.08.2017

http://www.salom.com.tr/, 22.08.2017 